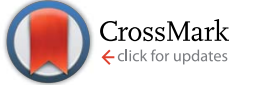

Cite this: Chem. Sci., 2017, 8, 3676

\title{
Structure and conserved function of iso-branched sphingoid bases from the nematode Caenorhabditis elegans $\dagger$
}

\author{
J. Thomas Hannich, ${ }^{\text {ac }}$ Denia Mellal,,$^{\text {bc }}$ Suihan Feng, ${ }^{\text {ac }}$ Andreas Zumbuehl $\S^{\star b c}$ \\ and Howard Riezman $\S^{\star a c}$
}

Sphingolipids are bio-active metabolites that show structural diversity among eukaryotes. They are essential for growth of all eukaryotic cells but when produced in an uncontrolled manner can lead to cell death and pathologies including auto-immune reactions, cancer, diabetes and neurodegeneration. Caenorhabditis elegans is an important genetic model organism both to find new drug-targets against parasitic nematodes and to study the conserved roles of sphingolipids in animals like their essential functions in very basic cellular processes ranging from maintenance of cell polarity and mitochondrial repair to growth and survival. C. elegans produces sphingoid bases which are structurally distinct from those of other animals as both iso- and anteiso-branched species have been reported. Using metabolic labeling we show that most worm sphingoid bases are iso-branched. We have synthesized the nematodespecific C17 iso-branched sphinganine and its 1-deoxy analogue and could show that both the isobranch and the 1-hydroxyl group are essential to form functional nematode sphingolipids which are needed to maintain intestinal function. The organism specificity was examined by complementation experiments in Saccharomyces cerevisiae yeast cells lacking sphingoid base synthesis. We found that iso-branched sphingoid base did not support growth of mutant cells and was toxic to wild type yeast. 1-Deoxy sphingolipids have been linked to the hereditary disease HSAN1A and other metabolic disorders including diabetes. We found that in C. elegans the 1-deoxy analogue cannot rescue the intestinal phenotype caused by sphingoid base depletion. In fact, in wild-type animals with normal sphingoid base biosynthesis, exogenous 1-deoxy analogue had a disruptive effect on apical cytoskeletal organization of intestinal cells indicating that atypical bases can interfere with normal sphingolipid function.

Received 31st October 2016 Accepted 3rd March 2017

DOI: $10.1039 / \mathrm{c} 6 \mathrm{sc} 04831 \mathrm{e}$

rsc.li/chemical-science

\section{Introduction}

Sphingolipids are one of the major eukaryotic membrane lipids and show species-specific structural differences. ${ }^{1}$ Long-chain bases, the central building block of all sphingolipids, can vary in their chemical structure with mainly straight-chain C18 bases in mammals and the yeast Saccharomyces cerevisiae, shorter C14 and C16 bases in flies, ${ }^{2,3}$ while for nematodes there have been reports of both $\mathrm{C} 17$ iso-branched ${ }^{4}$ and $\mathrm{C} 17$ anteisobranched bases. ${ }^{5}$ Branched-chain sphingoid bases have also been reported for Ascaris suum, ${ }^{6}$ a parasitic nematode, which

${ }^{a}$ Department of Biochemistry, University of Geneva, CH-1205 Geneva, Switzerland. E-mail: howard.riezman@unige.ch

${ }^{b}$ Department of Chemistry, University of Fribourg, CH-1700 Fribourg, Switzerland. E-mail: andreas.zumbuehl@unifr.ch

${ }^{c}$ National Centre of Competence in Research (NCCR) “Chemical Biology”, Switzerland $\dagger$ Electronic supplementary information (ESI) available: Experimental procedures and supplementary figures. See DOI: 10.1039/c6sc04831e

\$ Current address: MRC Laboratory of Molecular Biology, Cambridge Biomedical Campus, Cambridge CB2 0QH, UK.

$\S$ Equal contribution. makes the nematode-specific sphingolipid pathway a potential drug target. The initial step of sphingoid base biosynthesis is catalysed by the pyridoxal phosphate-dependent serine palmitoyl transferase (SPT) complex, which transfers serine to a fatty acylcoenzyme A and decarboxylates it to form 3-ketosphinganine that is further reduced to sphinganine. ${ }^{7}$ SPT highly prefers serine, but can also transfer alanine and glycine. ${ }^{8}$ In Hereditary Sensory and Autonomic Neuropathy Type-1A (HSAN1A) the SPT enzyme discrimination against alanine is reduced giving rise to increased amounts of 1-deoxysphinganine, which causes neurodegeneration, skin lesions, and muscle weakness. ${ }^{9}$ Increased amounts of 1-deoxysphinganine are also seen in metabolic disorders, including diabetes ${ }^{\mathbf{1 0}}$ and metabolic syndrome. ${ }^{11}$ Further structural diversity in sphingoid bases is achieved by desaturations and hydroxylations. ${ }^{3}$ While $S$. cerevisiae predominantly hydroxylates sphinganine (d18:0) to give rise to 4-hydroxysphinganine (t18:0), the major modification in mammals and worms is a desaturation at position 4 of the respective sphinganines (d18:0 in mammals and branched d17:0 in worms) resulting in sphingosine (d18:1) and C17 branched-chain sphingosine (branched d17:1), respectively (see Fig. 1b). ${ }^{3}$ Genetic studies 
a

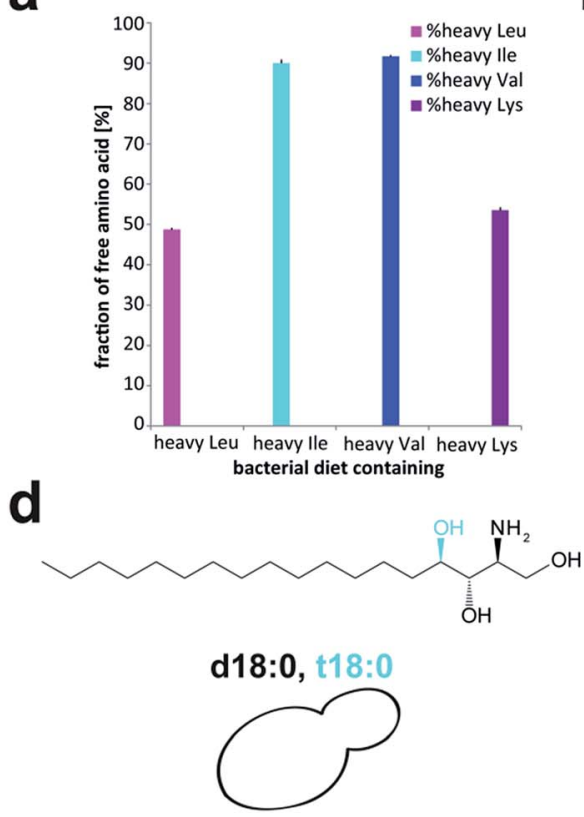

b
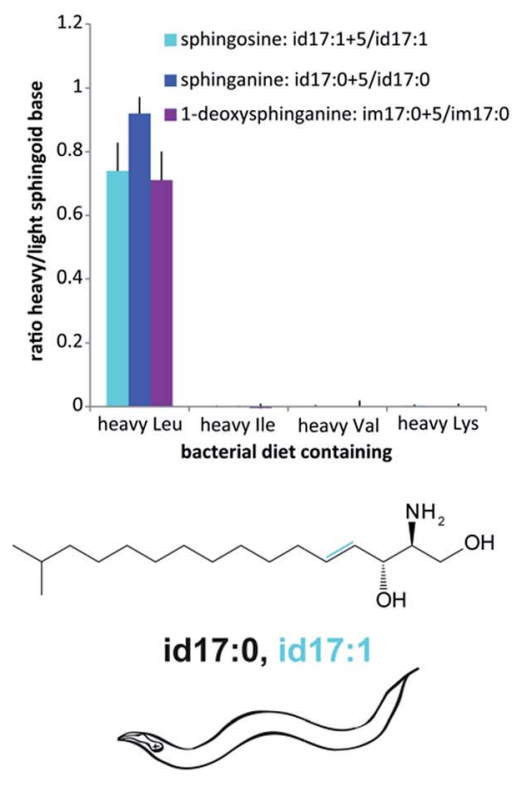

C

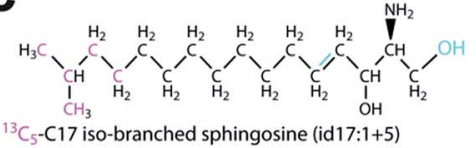

$\mathrm{C}_{5}-\mathrm{C} 17$ iso-branched sphingosine (id 17:1+5)

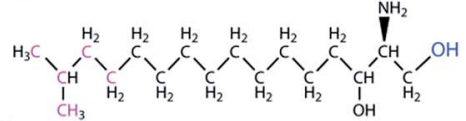

${ }^{13} \mathrm{C}_{5}-\mathrm{C} 17$ iso-branched sphinganine (id17:0+5)

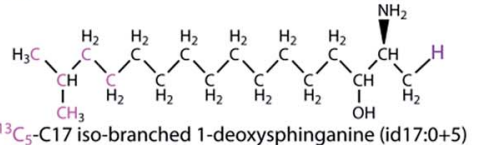<smiles>CCCCCCCCCCCCCCCC(O)C(N)CO</smiles>

d18:0, d18:1

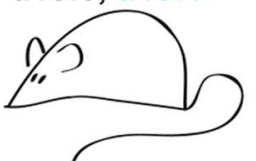

Fig. 1 The nematode C. elegans has mostly C17 iso-branched sphingoid bases. (a) Metabolic labelling of nematodes with heavy isotope leucine, isoleucine, valine, and lysine represented as percentage heavy of total free amino acid (average of $n=4)$, error bars show standard deviation; (b) incorporation of heavy isotope label from amino acids into sphingoid bases after isotopic peak correction (average of $n=4$ ), error bars show standard deviation; (c) structures of detected worm sphingoid bases with incorporated ${ }^{13} \mathrm{C}$ label, see also Scheme 1 and ESI Fig. $1 \mathrm{c} \dagger$ (d) major sphingoid bases from budding yeast: sphinganine (d18:0) and 4-hydroxysphinganine (t18:0, changes in cyan), from nematodes: C17 isobranched sphinganine (id17:0) and C17 iso-branched sphingosine (id17:1 changes in cyan), from mammals: sphinganine (d18:0) and sphingosine (d18:1, changes in cyan); shorthand nomenclature for sphingoid bases "a $X X: Y$ ": a-number of hydroxyl groups $(m=1, d=2, t=3), X X$-number of carbon atoms, $Y$-number of double bonds, the prefix " $i$ " indicates the iso-branch.

in C. elegans have revealed essential roles for sphingolipids in important cellular processes such as anoxia resistance, ${ }^{\mathbf{1 2}}$ apoptosis ${ }^{13}$ and mitochondrial surveillance. ${ }^{14}$ In addition, sphingolipids play an important developmental role in intestinal structure formation and function. ${ }^{15}$ While the functional conservation of sphingolipid biology has been confirmed in higher animals ${ }^{16}$ more detailed mechanistic studies on the physiological roles of sphingolipids in worms have been hindered by the absence of readily available $\mathrm{C} 17$ iso-branched sphingoid bases. Chemical genetic studies in $C$. elegans that help to elucidate the basic molecular mechanisms of sphingolipid physiology will lead to a deeper insight into sphingolipid physiology in higher animals including related processes in humans. While 1-deoxysphinganine has been identified as the causative metabolite leading to neurodegeneration in HSAN1A, ${ }^{9}$ the mechanism of action and possible molecular targets of the compound are not well understood. Production of 1-deoxysphinganine has been confirmed in humans, rats, mice, flies, and other animals ${ }^{7,17}$ but the possible presence and biological activities in C. elegans have not yet been studied.

\section{Results and discussion}

\section{Metabolic labelling of Caenorhabditis elegans sphingoid bases}

The C. elegans SPT complex synthesizes branched sphingoid bases from branched acyl-CoAs, ${ }^{18}$ which are known to be derived from branched-chain amino acids (BCAA) both in mammals ${ }^{19}$ and worms. ${ }^{20}$ In order to clarify the position of the branch point in $C$. elegans sphingoid bases, we fed animals for three generations with bacterial diets containing different heavy isotope-labelled amino acids: iso-branched ${ }^{13} \mathrm{C}_{6}$, L-leucine and ${ }^{13} \mathrm{C}_{5},{ }^{15} \mathrm{~N}$, L-valine, anteiso-branched ${ }^{13} \mathrm{C}_{6}$, L-isoleucine, and straight-chain ${ }^{13} \mathrm{C}_{6},{ }^{15} \mathrm{~N}_{2}$, L-lysine as a control. BCAA lose the amino nitrogen and one carbon atom during degradation to branched-chain fatty acids (see Scheme 1 and ESI Fig. 1c $\dagger$ ) and after fatty acid elongation a maximum of five heavy carbons of heavy leucine and isoleucine could be incorporated into sphingoid bases. All four heavy amino acids were readily taken up by the worms and were detected as free amino acids in worm lysates. In the case of isoleucine and valine, the exogenous heavy compound almost entirely replaced the endogenous light one. In our system no interconversion from one heavy amino acid to another was observed (Fig. 1a). We could detect C17 sphinganine, sphingosine and 1-deoxysphinganine in C. elegans extracts with $\mathrm{C} 17$ sphinganine being the most abundant one (ESI Fig. 1a $\dagger$ ). After correction for isotopic distribution (see Experimental) we could only detect +5 heavy sphingoid bases above background when feeding worms with heavy leucine (Fig. 1b and c). We conclude, therefore, that almost all branched sphingoid bases produced from BCAA by C. elegans carry an iso-branch. 
<smiles>CC[C@H](C)[C@@H](N)C(=O)O</smiles><smiles>CC(C)C[C@@H](N)C(=O)O</smiles>

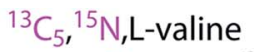

Branched-chain $\alpha$-ketoacid

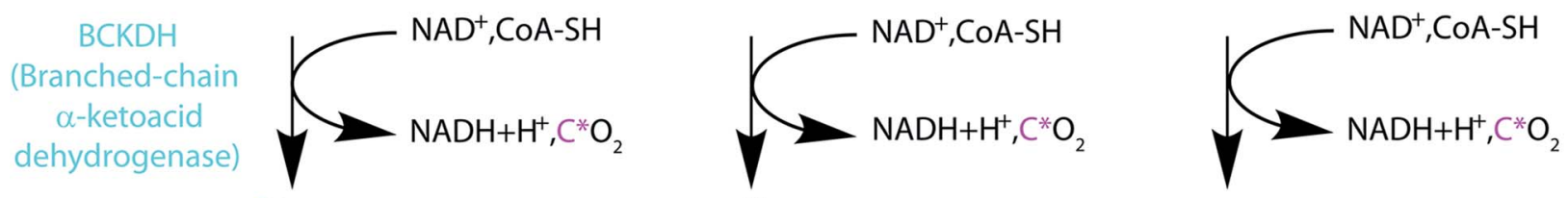<smiles>CC[C@H](C)C(=O)SO</smiles><smiles>C1CCCCC1</smiles>

$\mathrm{H}_{2}$<smiles>CC(C)C(=O)SO</smiles>

Fatty acid elongation cycles<smiles>CC(C)CCCCCCCCCCCC(=O)O[Na]</smiles>

C17 iso-branched 3-Ketosphinganine $+\mathrm{CO}_{2}$

C17 iso-branched 3-Keto-1-deoxysphinganine $+\mathrm{CO}_{2}$<smiles>CCCCCCCCCCCCC(O)[C@@H](N)CO</smiles>

${ }^{13} \mathrm{C}_{5}$ - $\mathrm{C} 17$ iso-branched sphinganine (id17:0)

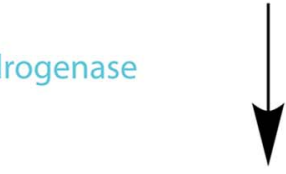<smiles>CCCCCCCCC(O)[C@@H](C)N</smiles>

${ }^{13} \mathrm{C}_{5}$ - $\mathrm{C} 17$ iso-branched 1-deoxysphinganine (im17:0)

Scheme $1{ }^{13} \mathrm{C}$ heavy isotope label incorporation from branched-chain amino acids (BCAAs) into $\mathrm{C} 17$ iso-branched sphingoid bases. BCAAs are converted into branched-chain acyl-coenzyme $\mathrm{A}(\mathrm{COA})$ derivatives by transamination using $\alpha$-ketoglutarate $(\alpha \mathrm{KG})$ and oxidative decarboxylation by branched-chain $\alpha$-ketoacid dehydrogenase $(\mathrm{BCKDH})$ which leads to loss of one ${ }^{13} \mathrm{C}$ label as $\mathrm{CO}_{2}$. Several cycles of fatty acid elongation give rise to $\mathrm{C} 15$ branched-chain acyl-CoA. In C. elegans serine palmitoyl-CoA transferase (SPT) transfers serine or alanine onto C15 iso-branched acyl-CoA to produce iso-branched 3-ketosphinganine and 3-keto-1-deoxysphinganine which are subsequently reduced to $\mathrm{C} 17$ iso-branched sphinganine and 1-deoxysphinganine. Only iso-branched derivatives of L-leucine are incorporated into nematode sphingoid bases.

\section{Chemical synthesis of C17 iso-branched sphingoid bases}

In order to study the function of sphingoid bases in C. elegans we developed a straight-forward synthesis of the two de novo synthesis sphingoid base products, $\mathrm{C} 17$ iso-branched sphinganine (id17:0) and its 1-deoxy analogue (im17:0) inspired by the work of Ha and Lee and coworkers (Fig. 2a). ${ }^{21}$ Both synthetic routes started with the commercially available precursor $(2 S)-2$ isopropyl-4-methoxycyclohexyl 1-((R)-1-phenylethyl)aziridine-2carboxylate 1 (Fig. 2a). The Weinreb amide 2 was installed following a literature protocol. ${ }^{22}$ The amide 2 was then substituted by a Grignard reagent 4 prepared from 


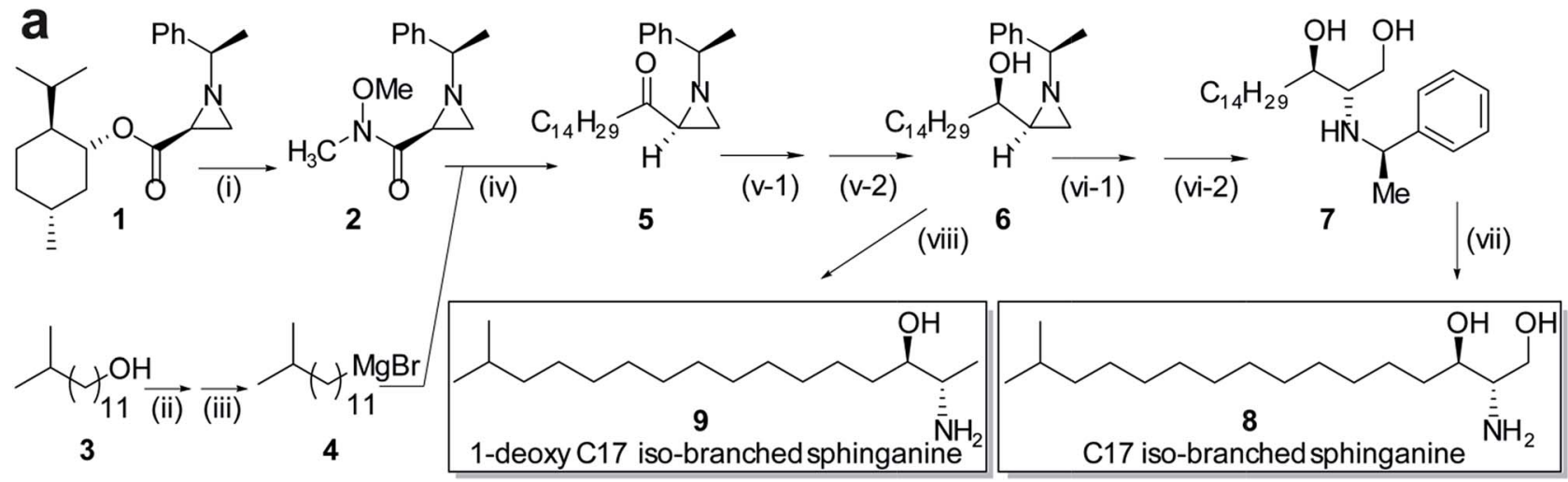

b

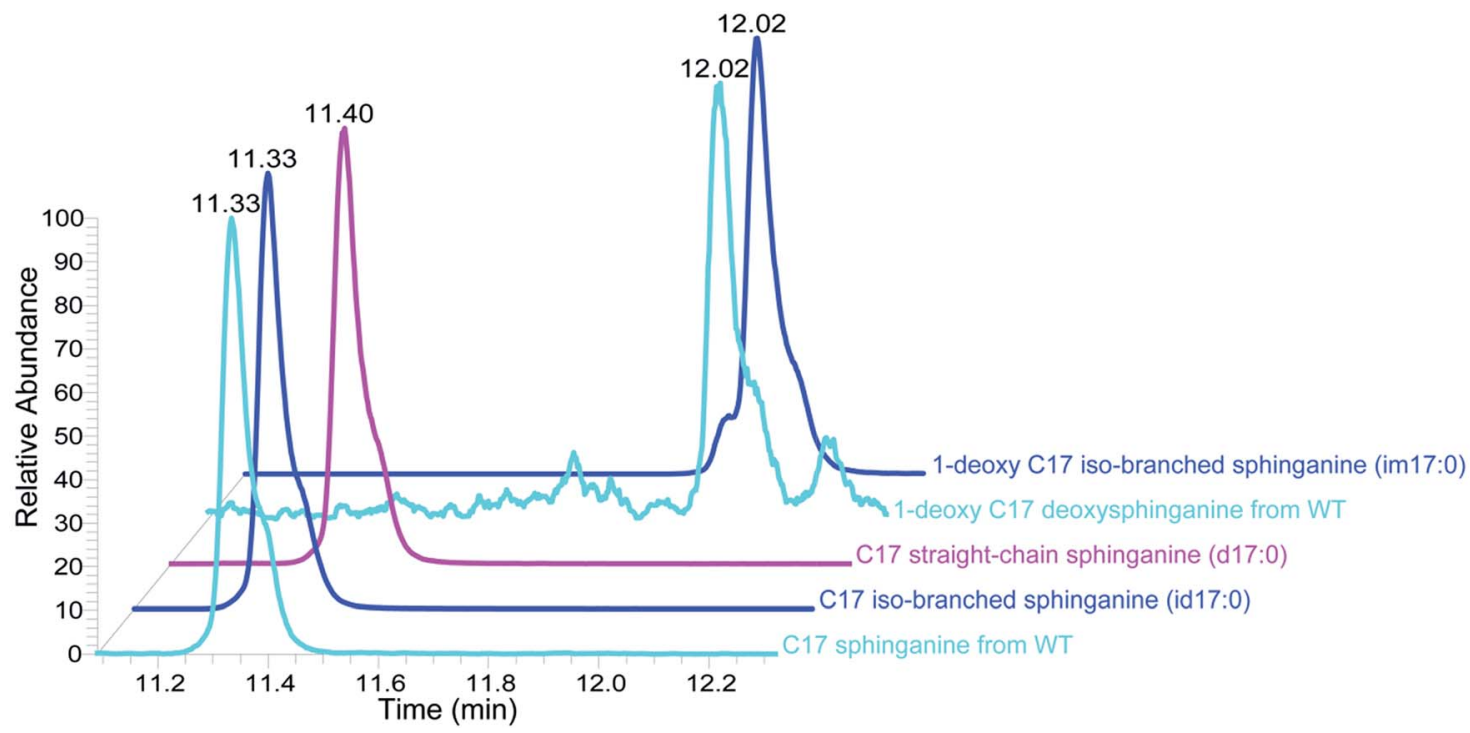

Fig. 2 C17 iso-branched sphingoid bases as analytical standards. (a) Synthetic route for nematode $\mathrm{C} 17$ iso-branched sphinganine (8) and its 1 deoxy analogue (9): (i) Weinreb's amine, $\mathrm{AlMe}_{3}, \mathrm{CH}_{2} \mathrm{Cl}_{2},-10{ }^{\circ} \mathrm{C}, 30 \mathrm{~min}$, then rt, $2 \mathrm{~h}, 45 \%$; (ii) $\mathrm{PPh}_{3} / \mathrm{Br}_{2}, \mathrm{pyridine} / \mathrm{CH}_{2} \mathrm{Cl}_{2}, 3 \mathrm{~h}, \mathrm{rt}, 88 \%$; (iii) $\mathrm{Mg}$, I 2 , dry THF, $20 \mathrm{~min}, 66^{\circ} \mathrm{C}$, quantitative yield; (iv) THF, $-78{ }^{\circ} \mathrm{C}, 30 \mathrm{~min}, 45 \%$; (v-1) $\mathrm{ZnCl}, \mathrm{MeOH}_{1}-78{ }^{\circ} \mathrm{C}, 30 \mathrm{~min}$; (v-2) $\mathrm{NaBH}_{4}, 30 \mathrm{~min},-78{ }^{\circ} \mathrm{C}, 80 \%$; (vi-1) $\mathrm{AcOH}, \mathrm{CH}_{2} \mathrm{Cl}_{2}, \mathrm{rt}, 18 \mathrm{~h}$, (vi-2) $\mathrm{KOH}, \mathrm{EtOH}, \mathrm{rt}, 1.5 \mathrm{~h}$; (vii) $\mathrm{H}_{2}, \mathrm{Pd} / \mathrm{C}, \mathrm{MeOH}$, rt, overnight, 58\%; (viii) $\mathrm{H}_{2}, \mathrm{Pd}(\mathrm{OH})_{2} / \mathrm{C}, \mathrm{MeOH}, \mathrm{rt}, \mathrm{overnight}, 42 \%$; (b) LCMS profiles of endogenous C17 sphinganine and 1-deoxy C17 sphinganine from WT C. elegans, synthesized iso-branched C17 bases, and commercial C17 straight-chain sphinganine. Retention times are indicated above each peak.

12-methyltridecan-1-ol 3 to give the corresponding ketone 5. The chelation-controlled reduction was further performed in the presence of $\mathrm{ZnCl}_{2}$ as bidentate chelating agent in order to give the corresponding aziridine alcohol $\mathbf{6}$. The aziridine ring $\mathrm{C}-\mathrm{N}$ bond was then reduced and a hydrogenation in the presence of Pearlman's catalyst provided the targeted sphinganineanalog 8. A hydrogenation using Pearlman's catalyst directly on the aziridine alcohol 6 provided the 1-deoxysphinganine analog 9 in one step (see Fig. 2a). The identity of the naturally occurring nematode sphingoid bases and the synthetic $\mathrm{C} 17$ iso-branched sphingoid bases was tested by LC-MS/MS. The C17 sphinganine extracted from wild-type nematodes had an identical retention time as the synthetic iso-branched molecule, but it ran clearly differently from a commercial C17 straight-chain isomer (Fig. 2b). C17 isobranched 1-deoxysphinganine could be detected in WT extracts, though, at much lower levels as can be seen by the increased background signal, and it showed the same retention time as the synthetic 1-deoxy C17 iso-branched sphinganine (Fig. 2b). This is the first time that a 1- deoxysphingolipid has been described in C. elegans and it demonstrates the importance of having synthetic compounds available to use as standards in LC-MS. No other C17 sphinganine or C17 1-deoxysphinganine signals above background were detected which makes the presence of significant amounts of other isomers in C. elegans unlikely.

\section{C. elegans digestion strictly depends on iso-branched sphingoid bases}

Sphingolipids are essential for the formation of functional intestines in C. elegans..$^{15}$ As a consequence worms with defects in glucosylceramide production, including any upstream biosynthetic step show impaired intestinal structure and digestive function. ${ }^{23}$ It has been previously reported that the second generation after knock-down of sphingolipid biosynthetic genes shows a larval arrest and starves. ${ }^{15}$ When the worm SPT subunit encoded by the sptl-1 gene was down-regulated via RNAi we observed, already in the first generation, that animals 
a

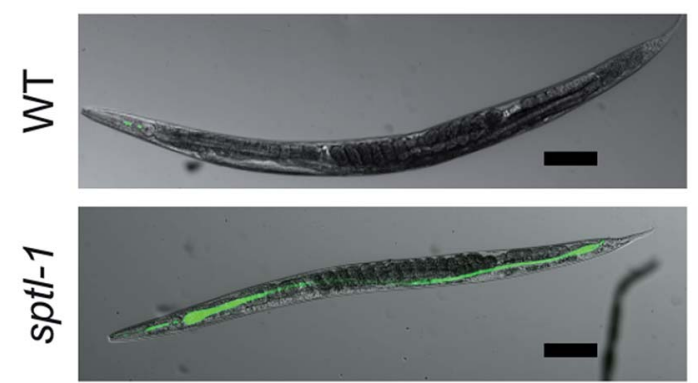

b

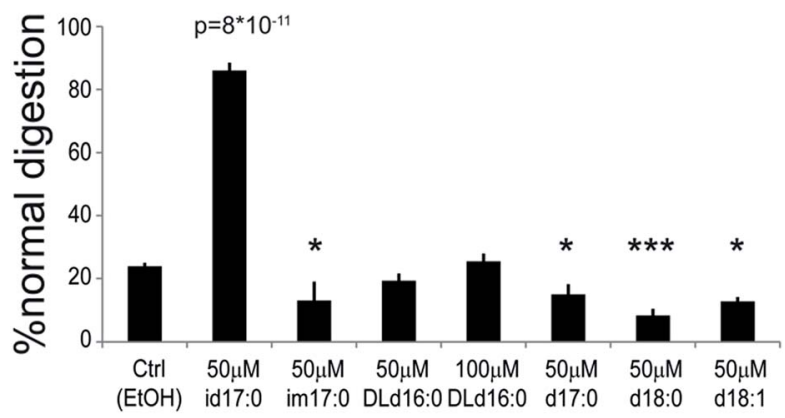

Fig. 3 C. elegans depends strictly on C17 iso-branched sphinganine for intestinal function. (a) Representative confocal images of wild-type and sptl-1 (RNAi) digestion phenotype after 20 minutes incubation, merge of transmitted light image of the animals (grey) and GFP channel showing OP50 GFP bacteria (green), scale bar = $100 \mu \mathrm{m}$; (b) quantification of the digestion phenotype of sptl-1 (RNAi) animals fed with control (EtOH), C17 iso-branched sphinganine (id17:0), C17 isobranched 1-deoxysphinganine (im17:0), racemic DL-C16 (DLd16:0), C17 straight-chain sphinganine (d17:0), sphinganine (d18:0), and sphingosine (d18:1). Normal digestion was scored as no detectable remaining GFP signal above background in the worm intestine. Error bars are standard errors for $n=6$ to 18 replicates, significance determined by Student's t-test: $* p<0.05, * * * p<0.005$.

were no longer capable of normally digesting GFP-expressing OP50 E. coli bacteria. ${ }^{24}$ A wild-type animal can normally digest all GFP signal in its intestine within 20 minutes while almost $80 \%$ of RNAi treated animals showed an accumulation of the fluorescent protein in the intestinal lumen (Fig. 3a). To investigate the significance of the iso-branch in nematode sphingoid bases we attempted to rescue the digestion phenotype by feeding the RNAi-treated nematodes with different sphingoid bases. Digestive function was only restored upon feeding $\mathrm{C} 17$ iso-branched sphinganine (id17:0, Fig. 3b). The 1-deoxy C17 iso-branched sphinganine (im17:0) was not capable of restoring normal digestion and even increased the congestion consistent with the requirement for the 1-hydroxyl group to form glucosylceramide. ${ }^{15}$ We also tested straight-chain C16, C17, and C18 sphingoid bases. Neither racemic C16 DL-straight-chain (DL-d16:0), C17 straight-chain (d17:0), C18 straight-chain sphinganine (d18:0), nor C18 straight-chain sphingosine (d18:1) were able to rescue the digestive function (Fig. $3 \mathrm{~b}$ ). In the case of straight-chain C17 sphinganine (d17:0), C18 sphinganine (d18:0) and sphingosine (d18:1) the digestion defect was even

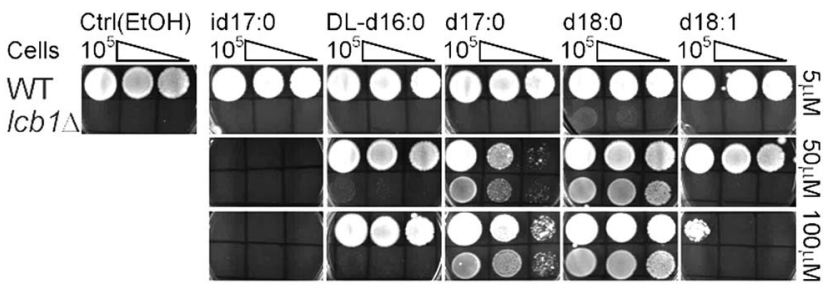

Fig. 4 C17 iso-branched sphinganine is toxic for budding yeast. Dilution spot assay of wild-type and $l c b 1 \Delta$ yeast cell growth on YPUATD plates with $0.05 \%$ tergitol and control vehicle (ethanol), C17 iso-branched sphinganine (id17:0), racemic DL-C16 (DL-d16:0), C17 straight-chain sphinganine (d17:0), sphinganine (d18:0), and sphingosine (d18:1) at 5, 50,100 $\mu \mathrm{M}$ concentration.

more severe. The observed dependency on iso-branched sphingoid bases for C. elegans development is in good agreement with recent findings that showed the importance of iso-branched fatty acid precursors for sphingolipid biosynthesis and to support development ${ }^{25,18}$. Therefore, the synthetic C17 iso-branched sphinganine is a valuable tool for researchers to test sphingoid base dependency of observed mutant and knock-down phenotypes. This clearly shows that the iso-branch structure in the C. elegans sphingoid base isomer compared to straight-chain base is functionally relevant. Recently, several genome-wide RNAi screens have identified sphingolipid biosynthetic genes involved in apical polarity formation ${ }^{23,26}$ and mitochondrial homoeostasis. ${ }^{14}$ Our synthetic bases can be used to complement other gene knock-downs with phenotypes similar to sphingolipid depletion in order to identify new sphingolipid regulators and effectors.

\section{Iso-branched sphingoid base is toxic for Saccharomyces cerevisiae}

In order to address the possible compatibility of the iso-branch in the nematode sphingoid base with other organisms, we conducted rescue experiments in an $S$. cerevisiae mutant defective in serine palmitoyltransferase $(l c b 1 \Delta)$. We supplied the mutant with different sphingoid bases in the growth media. Yeast $l c b 1 \Delta$ cells cannot grow on rich medium containing only tergitol and the carrier solvent, but are capable of growing on medium supplemented with C18 straight-chain sphinganine (d18:0), a product of its endogenous de novo sphingoid base biosynthetic pathway, and also on C17 straight-chain sphinganine (d17:0), and even to some extent on racemic C16 straightchain DL-sphinganine (DL-d16:0) (Fig. 4 and ESI Fig. $3 \dagger$ ). Sphingosine (d18:1), which is the major sphingoid base in mammals, does not support growth of $l c b 1 \Delta$ cells and shows toxicity at very high concentrations, consistent with previous work on sphingosine effects on budding yeast. ${ }^{27,28}$ Interestingly, the C17 iso-branched sphinganine isomer (id17:0) does not support growth and is toxic to WT yeast cells at lower concentrations than sphingosine (Fig. 4). While wild-type yeast cells readily supported $50 \mu \mathrm{M}$ sphingosine they cannot grow on 50 $\mu \mathrm{M}$ C17 iso-branched sphinganine (Fig. 4). 
a

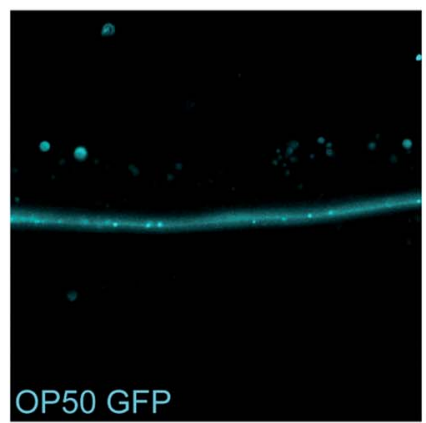

b

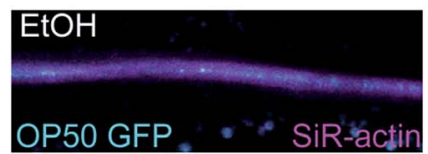

C

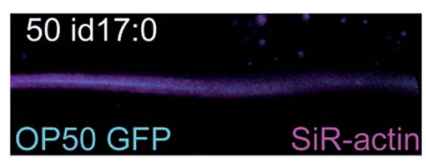

f

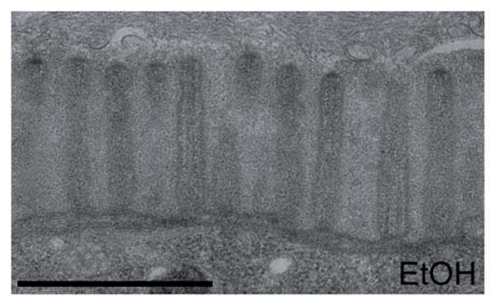

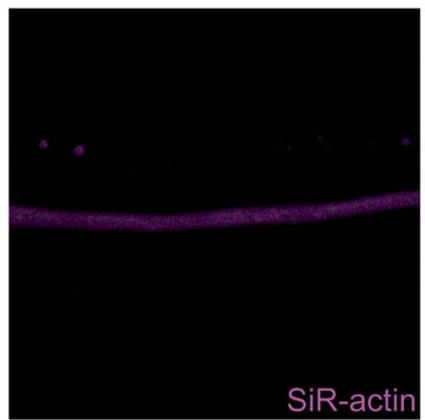

d
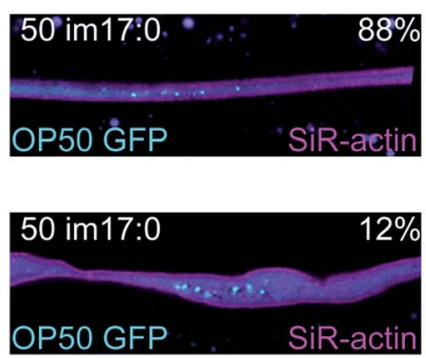

g

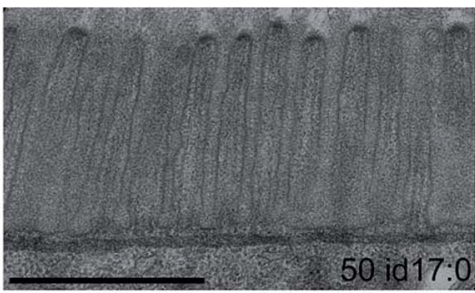

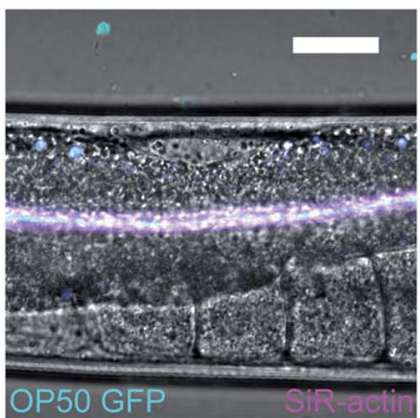

e

h
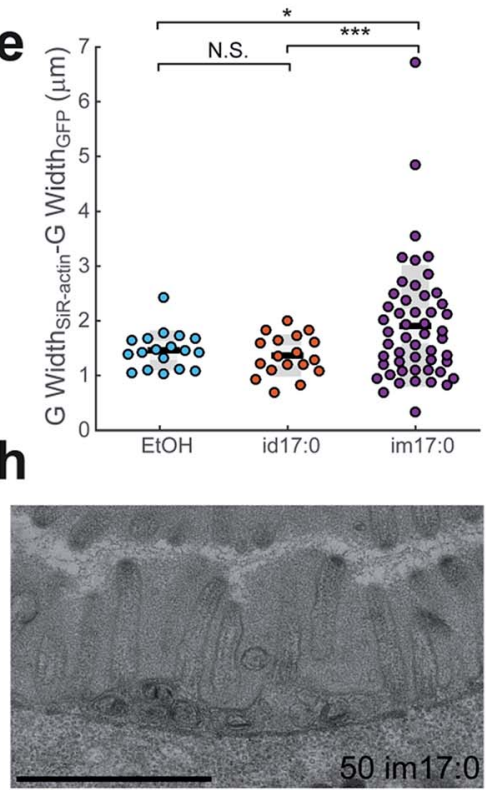

Fig. 5 1-Deoxy C17 iso-branched sphinganine causes changes in the apical brush border of $C$. elegans intestinal cells. (a) Confocal images of animals fed with OP50 GFP bacteria, SiR-actin probe and control ethanol vehicle (EtOH), OP50 GFP bacteria (cyan), SiR-actin (magenta), merge of fluorescence channels with transmitted light (grey), white scale bar $20 \mu \mathrm{m}$; (b-d) representative merged confocal images of OP50 GFP (cyan) and SiR-actin (magenta) signals in animals treated with ethanol vehicle (b), $50 \mu \mathrm{M}$ C17 iso-branched sphinganine, id17:0 (c) and 50 $\mu$ M 1-deoxy C17 iso-branched sphinganine, im17:0 (d); (e) width of apical F-actin belt as calculated by the difference between the Gaussian fits of the SiRactin and the GFP signals; statistical significance determined by Welch's $t$-test $* p<0.05, * * * p<0.005$; (f-h) representative electron micrographs showing the apical brush border of intestinal cells in animals treated with $\mathrm{EtOH}$ (f), $50 \mu \mathrm{M} \mathrm{C17} \mathrm{iso-branched} \mathrm{sphinganine,} \mathrm{id17:0} \mathrm{(g)} \mathrm{and} 50 \mu \mathrm{M} 1$ deoxy C17 iso-branched sphinganine, im17:0 (h), black scale bars represent $1 \mu \mathrm{m}$.

\section{1-Deoxy C17 iso-branched sphinganine affects F-actin organization in C. elegans intestinal brush border}

Accumulation of 1-deoxysphinganine in humans is associated with neurodegenerative symptoms as well as skin ulcerations and lower limb weakness. ${ }^{9}$ Some of the toxicity connected to 1deoxysphinganine observed in cell culture experiments is attributed to effects on the cytoskeleton, especially actin stress fibers. ${ }^{29}$ We raised wild type worms on NGM media containing either solvent control (EtOH), C17 iso-branched sphinganine (id17:0) or 1-deoxy C17 iso-branched sphinganine (im17:0) from L1 larval stage to adulthood. After 3 days, worms were transferred to plates seeded with GFP-expressing OP50 bacteria ${ }^{24}$ containing $3 \mu \mathrm{M} \mathrm{SiR-actin}{ }^{30}$ for 3 hours. SiR-actin is a turn-on probe that can be used for live-imaging of F-actin because it neither needs fixation nor washing. As worms were directly picked into anesthetizing M9 buffer containing $60 \mathrm{mM}$ sodium azide, GFP from the bacteria was not fully digested and the fluorescent signal was still detectable in the intestinal lumen (Fig. 5a, cyan). The specific F-actin signal (Fig. 5a, magenta) in WT animals fed with solvent control almost coincided with the intestinal lumen labelled by the GFP bacteria consistent with labelling of apical F-actin structures surrounding the lumen. Similar to control animals (EtOH, Fig. 5b) worms fed with $50 \mu \mathrm{M}$ C17 iso-branched sphinganine (50 id17:0, Fig. 5c) exhibited Factin signals in close proximity to the GFP positive intestinal lumen. In the case of animals grown on $50 \mu \mathrm{M}$ 1-deoxy C17 isobranched sphinganine (50 im17:0, Fig. 5d) the bacterial GFP signal (cyan) and the SiR-actin (magenta) signals coincided less. Effects of im17:0 on the worm intestines showed a certain variation in penetrance. While the majority of im17:0 treated animals exhibited a GFP-positive lumen width slightly wider on average than control conditions about $12 \%$ of the animals showed a severely widened intestinal lumen and accumulation of intact GFP bacteria. Therefore, in both cases the majority of im17:0 treated animals showed a clear separation of GFP and F- 

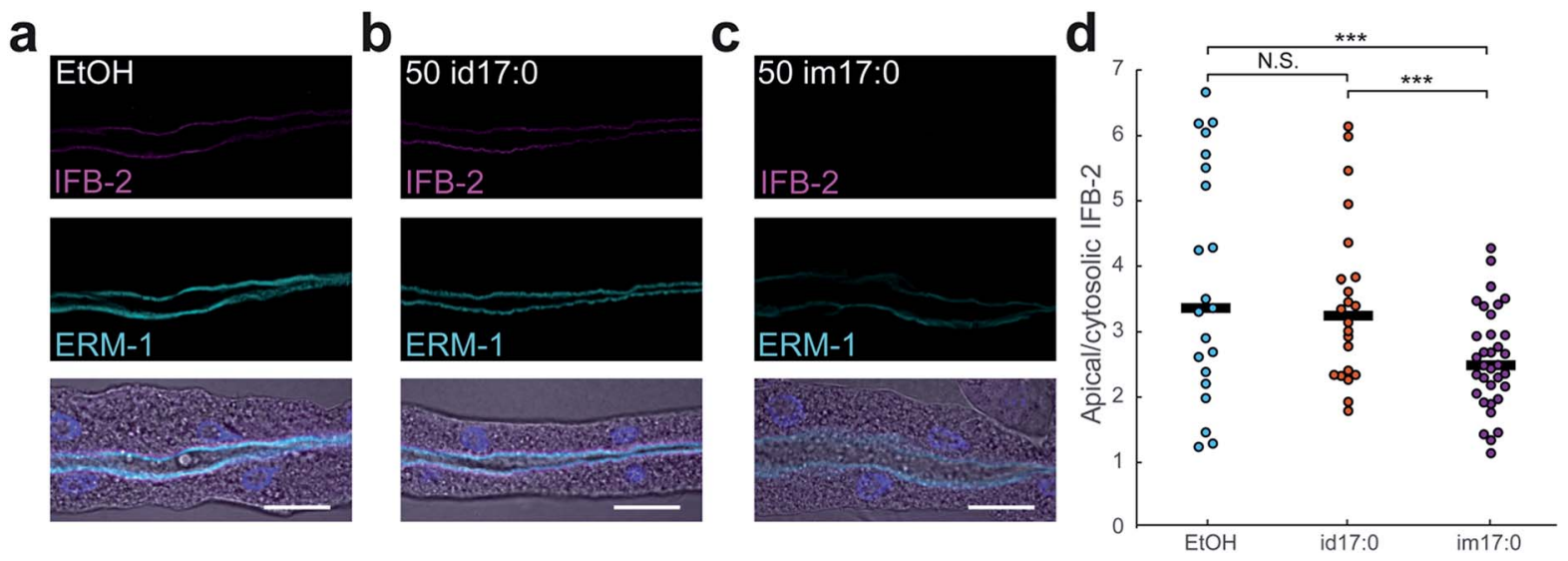

Fig. 6 1-Deoxy $\mathrm{C} 17$ iso-branched sphinganine disrupts terminal web of $C$. elegans intestinal cells through interference with intermediate filament formation. (a-c) Individual and merged confocal images of fixed intestines from animals fed with ethanol vehicle, EtOH (a), $50 \mu \mathrm{M} \mathrm{C17}$ iso-branched sphinganine, id17:0 (b), and $50 \mu \mathrm{M}$ 1-deoxy C17 iso-branched sphinganine, im17:0 (c), intermediate filament protein IFB-2 in magenta, ERM-1::GFP in cyan, DAPI in blue and DIC in grey, white scale bars represent $20 \mu \mathrm{m}$; (d) quantified signals of apical IFB-2 relative to cytosolic, statistical significance determined by Student's t-test: ***p $<0.005$.

actin signals. To quantify the morphological differences we recorded line profiles of the fluorescent signals perpendicular to the intestinal lumen together with the underlying endotube of intermediate filaments. The GFP signals were fitted by single Gaussian functions while a double Gaussian fit was used for the SiR-actin (ESI Fig. 2a and $b \dagger$ ). Average Gaussian curves for control conditions (EtOH, ESI Fig. 2c广), C17 iso-branched sphinganine (50 id17:0, ESI Fig. 2d $\dagger$ ), and 1-deoxy C17 isobranched sphinganine (50 im17:0, ESI Fig. $2 \mathrm{~d} \dagger$ ) show the widening of the intestinal lumen and the displacement of the Factin signal in the third case (ESI Fig. $2 \mathrm{~d} \dagger$ ). When the widths of the Gaussian fits were compared, a significant difference for the GFP positive lumen (ESI Fig. $2 \mathrm{f}^{\dagger}$ ) could be observed between id17:0 and im17:0 (Welsh's $t$-test $p=0.032$ ). The increase in width of the SiR-actin signals upon im17:0 treatment (ESI Fig. $2 \mathrm{~g} \dagger$ ) was even more significant (EtOH $v s$. im17:0 $p=0.011$, id17:0 vs. im17:0 $p=0.00088$ ) as well as the differences observed when looking only at the width of the apical F-actin as calculated by the difference between the widths of the SiR-actin and the GFP signals (Fig. 5e, EtOH vs. im17:0 $p=0.013$, id17:0 $v s$. im17:0 $p=0.0032$ ). Apical F-actin in intestinal cells is part of a very ordered cytoskeletal structure called the terminal web ${ }^{31}$ that serves to stabilize the intestinal lumen. Broadening of the F-actin signal indicates a loss of organization and might be the cause for deformation of the gut lumen in the more severe phenotype. An intestinal phenotype caused by sphingolipid depletion has been reported previously and it coincided with the actin organizer ERM-1 being delocalized from the apical membrane. ${ }^{23}$ We confirmed the changes of F-actin signal from light microscopy by electron microscopy. WT animals treated with control vehicle (EtOH, Fig. 5f) or $50 \mu \mathrm{M} \mathrm{C17}$ iso-branched sphinganine (50 id17:0, Fig. 5g) show a very ordered array of microvilli on the surface of their intestinal cells and a terminal web in close proximity to the plasma membrane but animals grown on $50 \mu \mathrm{M}$ 1-deoxy C17 iso-branched sphinganine (50 im17:0, Fig. 5h) showed irregular microvilli, discontinuous endotube intermediate filament structures below the plasma membrane and accumulated material between terminal web and plasma membrane. Effects on the cytoskeleton might be a very early event in the sphingolipid depletion phenotype that eventually leads to loss of polarity in intestinal cells and therefore loss of a continuous gut lumen. ${ }^{23}$

\section{1-Deoxy C17 iso-branched sphinganine interferes with intermediate filament formation}

Structure and stability of the intestinal brush border depend on the correct localization, organization and membrane association of F-actin as well as the underlying intermediate filaments of the endotube. Previously, it was shown that mutants in sphingolipid biosynthesis show a disorganized intestinal brush border associated with mis-localization of the F-actin organizer ERM-1 from apical to basolateral membrane. ${ }^{23}$ Baso-lateral expansion of apical ERM-1 coincides with baso-lateral expansion of apical intermediate filaments as well. ${ }^{32}$ We cultured ERM-1::GFP fusion protein expressing worms on media containing EtOH, $50 \mu \mathrm{M}$ id17:0, or $50 \mu \mathrm{M}$ im17:0 and observed no differences in ERM-1 expression (data not shown) as well as only apical localization under all conditions (Fig. 6a-c). When dissected intestines were immuno-stained for the intermediate filament component IFB-2 significant differences could be observed in animals grown on 1-deoxy C17 iso-branched sphinganine (50 im17:0) compared to the wild type animals grown on vehicle control (EtOH) and C17 iso-branched sphinganine (50 id17:0). While im17:0-fed worms still showed only apical membrane signals for IFB-2 (Fig. 6c) they were significantly weaker than EtOH control (Fig. 6a) and id17:0 (Fig. 6B) fed animals (Student's $t$-test: EtOH $v s$. id17:0 $p=0.49$, EtOH $v s$., $\operatorname{im} 17: 0 p=0.001, \operatorname{id} 17: 0$ vs. im17:0 $p=0.002 ; n=21-34)$. The phenotype in the apical brush border of the intestines is therefore not due to a mislocalization of F-actin organizers like ERM-1 but rather caused by a destabilization of the underlying intermediate filaments of the endotube. Loss of cytoskeletal 
structures including F-actin and intermediate filaments upon 1deoxysphinganine treatment has also been reported in cultured neuronal cells ${ }^{9,33}$ and might be the reason for many of the symptoms associated with the HSAN1 disease.

\section{Conclusions}

Studying sphingolipids in the genetic model organism C. elegans serves two important purposes: first, the structural peculiarity of the nematode sphingoid base makes sphingolipid biosynthesis a drug target to combat parasitic nematodes that infect plants, animals and humans. Second, the functional conservation of sphingolipid biology among animals makes it a valuable model to study stress responses, ageing, ${ }^{34}$ and development. ${ }^{15,16}$ Using stable isotope labelling we have addressed a long standing question in C. elegans lipid analysis whether worm sphingoid bases are iso-branched, ${ }^{4}$ anteisobranched or both. ${ }^{5}$ We show that the majority of free sphingoid bases in C. elegans are iso-branched. With our labelling technique we could not detect any anteiso-branched sphingoid bases. We cannot exclude the remote possibility that anteisobranched sphingoid bases could be produced by another yet unknown mechanism or under different culture conditions. If produced, though, they are definitely not required for development as the iso-branched sphinganine alone can rescue absence of endogenous sphingoid base production. ${ }^{18}$ This also explains recently published data that in mutants lacking BCAA degradation the developmental arrest can only be rescued by iso-branched $\mathrm{C} 5$ fatty acid but not by the anteiso-branched isomer because only the iso-branched one will be incorporated into the sphingoid base required for development. ${ }^{20}$ Isobranched sphingoid bases are known from animal-parasitic nematodes where biological material is available in large quantities. $^{6}$ The sensitive stable isotope labelling strategy described here can be used to elucidate sphingoid base structures in plant parasitic nematodes where it is very difficult to obtain sufficient material for classical structural analysis. Our newly developed synthesis procedure for nematode-specific C17 iso-branched sphinganine and its 1-deoxy analogue have served to show that the structural diversity of sphingoid bases is very important for their essential biological functions both in nematodes and yeast. Complementation experiments in C. elegans show the importance of the iso-branch for nematode development as straight-chain sphingoid bases are incompatible with nematode sphingolipid biosynthesis and function. This finding explains the mechanism of toxicity that was recently exploited to kill the parasitic nematode Meloidogyne incognita where production of straight-chain sphingoid bases by a soil bacterial strain was used to make tomato plants resistant to nematode infections. ${ }^{35}$ Our reverse complementation experiments in yeast cells underline the organism-specificity of sphingoid base structures as the C. elegans $\mathrm{C} 17$ iso-branched sphinganine is toxic to $S$. cerevisiae. Despite structural differences the functional conservation of sphingolipid biology from nematodes to higher animals is already evident in apoptosis, ${ }^{13}$ stress responses, ${ }^{12}$ and intestinal development. ${ }^{15,16}$ Our new findings on the conserved function of atypical 1-deoxysphingoid bases in disrupting cytoskeletal structures open the door to future studies in C. elegans that can further elucidate the mechanisms of toxicity of 1-deoxysphingolipids which have been connected to the hereditary disease HSAN ${ }^{9}$ and metabolic disorders like diabetes ${ }^{10}$ and metabolic syndrome. ${ }^{11}$

\section{Experimental}

\section{Material}

Heavy isotope labelled amino acids were purchased from Cambridge Isotope Laboratories Inc. (Andover, Massachusetts, USA), commercial sphingoid bases from Avanti Polar Lipids (Alabaster, Alabama, USA), except DL-C16-sphinganine from Matreya LLC (State College, Pennsylvania, USA), 12-methyl tridecanol from Endeavour Speciality Chemicals (Daventry, United Kingdom), SiR-actin from Spirochrome (Stein am Rhein, Switzerland), all other compounds and solvents at highest purity from Sigma-Aldrich (Buchs, Switzerland) or Acros Organics/Chemie Brunschwig (Basel, Switzerland), MH33 antibody was from DSHB (University of Iowa).

Wild-type N2 (var Bristol) and bacterial strains were received from the Caenorhabditis Genetics Centre (University of Michigan, Michigan, USA) VJ402 (fgEx11[ERM-1::GFP]) were a kind gift of Dr Verena Göbel. A complete list of bacteria, yeast strains and nematodes used in this study can be found in Table 1 . Unless stated otherwise bacteria were grown on LB medium, yeast on rich medium (YPUATD: $1 \%$ yeast extract, $2 \%$ bacto peptone, each $40 \mathrm{mg} \mathrm{l}^{-1}$ uracil, adenine, tryptophan, $2 \%$ glucose, $2 \%$ bacto agar) and worms on NGM medium seeded with OP50 bacteria. ${ }^{36}$

\section{Metabolic labelling of sphingoid bases with heavy isotope amino acids}

Bacterial diet (HT115 containing empty vector L4440) was grown to saturation in M63 minimal medium containing $40 \mu \mathrm{g}$ $\mathrm{ml}^{-1}$ of leucine, isoleucine, valine, and lysine. In each of the four different diet preparations one of the light amino acids was replaced by the corresponding amount of heavy isotope labelled compound. WT N2 animals were grown for three generations on nitrogen-free $10 \mathrm{~cm} \mathrm{NGM}$ plates $^{37}$ seeded with $300 \mu$ labelled bacteria ( $0.15 \mathrm{mg} \mathrm{ml}^{-1}$ suspension in M9 buffer). Animals were harvested in MS grade water, amino acids and sphingoid bases were extracted and analysed by LC-MS (see below). Heavy isotope containing +5 signals were corrected for isotopic peak contribution from unspecific +4 signals due to ${ }^{13} \mathrm{C}$ recycling from degradation and reincorporation via fatty acid elongation $^{19}$ (ESI Fig. 1a-b $\dagger$ ).

\section{Chemical synthesis of iso-branched sphingoid bases and LC- MS analysis}

A detailed description of the chemical synthesis of $\mathrm{C} 17$ isobranched sphinganine and 1-deoxy $\mathrm{C} 17$ iso-branched sphinganine can be found in the ESI. $\dagger$ LC-MS analysis was done according to Höglinger et al..$^{38}$ In short, sphingoid bases were resuspended in borate buffer $(200 \mathrm{mM}$ boric acid pH8.8, $10 \mathrm{mM}$ tris(2-carboxyethyl)phosphine, $10 \mathrm{mM}$ ascorbic acid and 
Table 1 Strains used in this study

\begin{tabular}{|c|c|c|}
\hline Name & Genotype & Source \\
\hline \multicolumn{3}{|l|}{ E. coli } \\
\hline OP50 & Uracil auxotrophic $E$. coli B (Berkley strain) & CGC \\
\hline HT115 (DE3) & F-, mcrA, mcrB, IN(rrnD-rrnE)1, lambda-, rnc14::Tn10(DE3 lysogen:lacUV5 promoter-T7 polymerase) & Kamath et al. 2003 \\
\hline HT115 sptl-1 & HT115 expressing plasmid L4440 (pPD129.36) containing a fragment of the sptl-1 cDNA & Kamath et al. 2003 \\
\hline RH1201 & 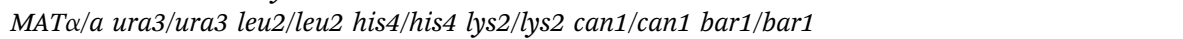 & This study \\
\hline RH6995 & 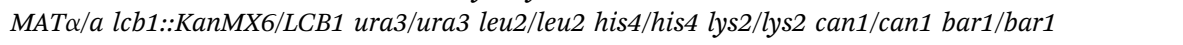 & This study \\
\hline RH6998 & MATa lcb1::KanMX6 ura3 leu2 his4 lys2 can1 bar1 & This study \\
\hline \multicolumn{3}{|l|}{ C. elegans } \\
\hline $\mathrm{N} 2$ (var. Bristol) & C. elegans wild isolate & Brenner S. 1974 \\
\hline
\end{tabular}

33.7 $\mathrm{mM}{ }^{13} \mathrm{C}^{15} \mathrm{~N}$-valine), derivatized with 6-aminoquinolylhydroxysuccinimidyl carbamate (AQC, $2.85 \mathrm{mg} \mathrm{ml}^{-1}$ in acetonitrile) at $55{ }^{\circ} \mathrm{C}$ for $15 \mathrm{~min}$ and left at $24{ }^{\circ} \mathrm{C}$ overnight. Samples were analyzed using a reverse-phase C18 column (HPLC EC 100/ 2 Nucleoshell RP-18 $2.7 \mathrm{~mm}$ ) on an Accela high-performance liquid chromatography system (ThermoFisher Scientific, Waltham, MA), coupled to a TSQ Vantage triple quadrupole mass spectrometer (ThermoFisher Scientific, Waltham, USA) using multiple reaction monitoring.

\section{Sphingoid base complementation of RNAi phenotype}

Ethanol and sphingoid bases at a concentration of 50 and 100 $\mu \mathrm{M}$ were applied and dried onto the surface of 12-well NGM agar plates containing $25 \mu \mathrm{g} \mathrm{ml}{ }^{-1}$ carbenicillin and $1 \mathrm{mM}$ IPTG. Plates were seeded with a $1: 1$ mix of OP50 GFP ${ }^{24}$ bacteria and HT115 (DE3) bacteria expressing an RNAi feeding vector from the Ahringer RNAi library targeting the sptl-1 gene. ${ }^{39}$ After overnight incubation at room temperature starved N2 L0 larvae were transferred to the wells and grown for three days at $20^{\circ} \mathrm{C}$. Young adults were washed off the plates with M9 buffer and collected in $1 \mathrm{ml}$ 96-well plates where they were washed once with M9 buffer. Twenty minutes after harvesting, worms were anaesthetized with M9 buffer containing $60 \mathrm{mM}$ sodium azide and transferred to a BD Falcon 96-well imaging plate. Imaging was performed on an ImageXpress ${ }^{\circledR}$ Micro XLS high-content microscope and image analysis was done with the help of MetaXpress ${ }^{\circledR}$ software. Statistical analysis was done using a Student's $t$-test.

\section{Sphingoid base effects on cytoskeleton}

Ethanol and sphingoid bases at a concentration of $50 \mu \mathrm{M}$ were applied and dried onto the surface of 12-well NGM agar plates. Plates were seeded with OP50 GFP bacteria containing $3 \mu \mathrm{M}$ SiRactin and $3 \mu \mathrm{M}$ verapamil. ${ }^{30}$ Plates were incubated overnight at room temperature. Starved N2 L0 larvae were transferred to the wells and grown for three days at $20{ }^{\circ} \mathrm{C}$. Young adults were picked directly into M9 buffer containing $60 \mathrm{mM}$ sodium azide and were imaged on a Zeiss LSM700 using a $63 \times$ objective. For image analysis three 16 to $18 \mu \mathrm{m}$ line profiles perpendicular to the intestinal lumen were measured per image using the Fiji software. Data fitting and statistical analysis was performed using a Matlab script. GFP signals were fitted to a single Gaussian function and width was determined as the full width at half maximum while the SiR-actin signal was fitted by a double Gaussian function and width was calculated as the distance between the two maxima plus half the sum of the two full widths at half maximum (ESI Fig. $2 \mathrm{a}$ and $\mathrm{b} \dagger$ ). Width of the apical F-actin belt was calculated as the difference between the SiR-actin and the GFP width. Statistical analysis was performed using Welsh's $t$-test.

For immunostaining with MH33 anitbody against IFB-2 ERM-1::GFP animals were grown for three days on media containing ethanol or sphingoid bases at a concentration of $50 \mu \mathrm{M}$, intestines were dissected in buffer containing $0.1 \%$ tricaine, and $0.001 \%$ levamisole. Fixation and immunostaining was performed according to standard procedures. ${ }^{26,32}$ For quantification of confocal images $20 \mu \mathrm{m}$ line profiles were taken across the intestinal lumen. Average apical signal was divided by average cytosolic. Student's $t$-test was used to compare the different conditions.

\section{Electron microscopy}

Animals were grown the same way as for light microscopy. Young adults were washed off plates using 0.1 M HEPES/NaOH pH 7.5, washed once with the same buffer and then with buffer containing $8 \%$ ethanol. Animals were cut and fixed in $2 \%$ glutaraldehyde and 4\% paraformaldehyde in $0.1 \mathrm{M}$ phosphate buffer $\mathrm{pH} 7.0$ for 4 hours at room temperature. Worms were washed three times in 0.1 M HEPES/NaOH pH 7.5, embedded in agarose and processed using standard procedures. ${ }^{\mathbf{4 0}}$ Images were recorded on a Tecnai G2 TEM at $100 \mathrm{keV}$.

\section{lcb1s Mutant strain construction}

In the diploid yeast strain RH1201, one complete open reading frame of $L C B 1$ was replaced with a KanMX6 cassette from the pFA6 plasmid via homologous recombination using primers 
ATGGCACACATCCCAGAGGTTTTACCCAAATCAATACCGATTCC GCGTACGCTGCAGGTCGAC and TAGATTCTTGGCAACAGGCA AGGATGGACTGCTTGACACACACTTATCGATGAATTCGAGCTCG to give rise to the heterozygous mutant RH6995. The haploid lcb1s strain RH6998 was isolated by tetrad dissection and its growth depends on exogenous sphingoid base supplementation $(10 \mu \mathrm{M})$ in the presence of $0.05 \%$ tergitol.

\section{Yeast growth assays}

Yeast strains were grown at $30^{\circ} \mathrm{C}$ overnight in liquid YPD with additional uracil, adenine, and tryptophan (YPUATD), in the $l c b 1 \Delta$ complementation experiment $0.05 \%$ tergitol NP-40 and $10 \mu \mathrm{M}$ sphinganine were added. Stationary yeast cultures were diluted to $\mathrm{OD}_{600}=1.5$ and consecutive ten-fold dilutions with double-distilled water. About $3 \mu \mathrm{l}$ of the dilutions were pinned onto YPUATD agar plates containing $0.05 \%$ tergitol NP-40, ethanol and different concentrations of sphingoid bases. Plates were incubated for two days at $30^{\circ} \mathrm{C}$.

\section{Acknowledgements}

We would like to thank France Favarger for help with the chemical synthesis, Isabelle Riezman for help with LC-MS analysis, Christoph Bauer, Jérôme Bosset, Pierre Cosson for fluorescence and electron microscopy support and Manuel Lera Ramírez for image and statistical analysis. Worms and bacterial strains were received from the Caenorhabditis Genetics Center, which is funded by NIH Office of Research Infrastructure Programs (P40 OD010440). JTH was supported by an EMBO/ Marie-Curie Long-Term Fellowship. This work was supported by grants from the Swiss National Science Foundation (SNSF) (to HR), the National Centre of Competence in Research (NCCR) Chemical Biology and SystemsX (evaluated by the SNSF) and the Canton of Geneva. AZ is supported by an SNSF assistant professorship.

\section{References}

1 S. T. Pruett, A. Bushnev, K. Hagedorn, M. Adiga, C. A. Haynes, M. C. Sullards, D. C. Liotta and A. H. Merrill Jr, J. Lipid Res., 2008, 49, 1621-1639.

2 H. Fyrst, D. R. Herr, G. L. Harris and J. D. Saba, J. Lipid Res., 2004, 45, 54-62.

3 J. T. Hannich, K. Umebayashi and H. Riezman, Cold Spring Harbor Perspect. Biol., 2011, 3, 189-202.

4 D. J. Chitwood, W. R. Lusby, M. J. Thompson, J. P. Kochansky and O. W. Howarth, Lipids, 1995, 30, 567-573.

5 S. Gerdt, G. Lochnit, R. D. Dennis and R. Geyer, Glycobiology, 1997, 7, 265-275.

6 G. Lochnit, R. D. Dennis, U. Zahringer and R. Geyer, Glycoconjugate J., 1997, 14, 389-399.

7 J. Duan and A. H. Merrill Jr, J. Biol. Chem., 2015, 290, 1538015389.

8 N. C. Zitomer, T. Mitchell, K. A. Voss, G. S. Bondy, S. T. Pruett, E. C. Garnier-Amblard, L. S. Liebeskind,
H. Park, E. Wang, M. C. Sullards, A. H. Merrill Jr and R. T. Riley, J. Biol. Chem., 2009, 284, 4786-4795.

9 A. Penno, M. M. Reilly, H. Houlden, M. Laura, K. Rentsch, V. Niederkofler, E. T. Stoeckli, G. Nicholson, F. Eichler, R. H. Brown Jr, A. von Eckardstein and T. Hornemann, J. Biol. Chem., 2010, 285, 11178-11187.

10 A. Othman, C. H. Saely, A. Muendlein, A. Vonbank, H. Drexel, A. von Eckardstein and T. Hornemann, BMJ Open Diabetes Res Care., 2015, 3, e000073.

11 A. Othman, M. F. Rutti, D. Ernst, C. H. Saely, P. Rein, H. Drexel, C. Porretta-Serapiglia, G. Lauria, R. Bianchi, A. von Eckardstein and T. Hornemann, Diabetologia, 2012, 55, 421-431.

12 V. Menuz, K. S. Howell, S. Gentina, S. Epstein, I. Riezman, M. Fornallaz-Mulhauser, M. O. Hengartner, M. Gomez, H. Riezman and J. C. Martinou, Science, 2009, 324, 381-384. 13 X. Deng, X. Yin, R. Allan, D. D. Lu, C. W. Maurer, A. Haimovitz-Friedman, Z. Fuks, S. Shaham and R. Kolesnick, Science, 2008, 322, 110-115.

14 Y. Liu, B. S. Samuel, P. C. Breen and G. Ruvkun, Nature, 2014, 508, 406-410.

15 E. Marza, K. T. Simonsen, N. J. Faergeman and G. M. Lesa, J. Cell Sci., 2009, 122, 822-833.

16 R. Jennemann, S. Kaden, R. Sandhoff, V. Nordstrom, S. Wang, M. Volz, S. Robine, N. Amen, U. Rothermel, H. Wiegandt and H. J. Grone, J. Biol. Chem., 2012, 287, 32598-32616.

17 A. Othman, R. Bianchi, I. Alecu, Y. Wei, C. PorrettaSerapiglia, R. Lombardi, A. Chiorazzi, C. Meregalli, N. Oggioni, G. Cavaletti, G. Lauria, A. von Eckardstein and T. Hornemann, Diabetes, 2015, 64, 1035-1045.

18 H. Zhu, H. Shen, A. K. Sewell, M. Kniazeva and M. Han, eLife, 2013, 2, e00429.

19 H. Oku, N. Yagi, J. Nagata and I. Chinen, Biochim. Biophys. Acta, 1994, 1214, 279-287.

20 F. Jia, M. Cui, M. T. Than and M. Han, J. Biol. Chem., 2016, 291, 2967-2973.

21 J. M. Yun, T. B. Sim, H. S. Hahm, W. K. Lee and H. J. Ha, The J. Org. Chem., 2003, 68, 7675-7680.

22 J. W. Kim, Y. W. Kim, Y. Inagaki, Y. A. Hwang, S. Mitsutake, Y. W. Ryu, W. K. Lee, H. J. Ha, C. S. Park and Y. Igarashi, Bioorg. Med. Chem., 2005, 13, 3475-3485.

23 H. Zhang, N. Abraham, L. A. Khan, D. H. Hall, J. T. Fleming and V. Gobel, Nat. Cell Biol., 2011, 13, 1189-1201.

24 A. Labrousse, S. Chauvet, C. Couillault, C. L. Kurz and J. J. Ewbank, Curr. Biol., 2000, 10, 1543-1545.

25 E. V. Entchev, D. Schwudke, V. Zagoriy, V. Matyash, A. Bogdanova, B. Habermann, L. Zhu, A. Shevchenko and T. V. Kurzchalia, J. Biol. Chem., 2008, 283, 17550-17560.

26 J. F. Winter, S. Hopfner, K. Korn, B. O. Farnung, C. R. Bradshaw, G. Marsico, M. Volkmer, B. Habermann and M. Zerial, Nat. Cell Biol., 2012, 14, 666-676.

27 G. B. Wells and R. L. Lester, J. Biol. Chem., 1983, 258, 1020010203.

28 S. Murakami, T. Shimamoto, H. Nagano, M. Tsuruno, H. Okuhara, H. Hatanaka, H. Tojo, Y. Kodama and K. Funato, Sci. Rep., 2015, 5, 16319. 
29 R. Cuadros, E. Montejo de Garcini, F. Wandosell, G. Faircloth, J. M. Fernandez-Sousa and J. Avila, Cancer Lett., 2000, 152, 23-29.

30 G. Lukinavicius, L. Reymond, E. D'Este, A. Masharina, F. Gottfert, H. Ta, A. Guther, M. Fournier, S. Rizzo, H. Waldmann, C. Blaukopf, C. Sommer, D. W. Gerlich, H. D. Arndt, S. W. Hell and K. Johnsson, Nat. Methods, 2014, 11, 731-733.

31 K. Carberry, T. Wiesenfahrt, F. Geisler, S. Stocker, H. Gerhardus, D. Uberbach, W. Davis, E. Jorgensen, R. E. Leube and O. Bossinger, Development, 2012, 139, 1851-1862.

32 O. Bossinger, T. Fukushige, M. Claeys, G. Borgonie and J. D. McGhee, Dev. Biol., 2004, 268, 448-456.

33 T. Guntert, P. Hanggi, A. Othman, S. Suriyanarayanan, S. Sonda, R. A. Zuellig, T. Hornemann and O. O. Ogunshola, Neuropharmacology, 2016, 110, 211-222.
34 X. Deng and R. Kolesnick, Biol. Chem., 2015, 396, 767-773.

35 H. Gao, G. Qi, R. Yin, H. Zhang, C. Li and X. Zhao, Sci. Rep., 2016, 6, 28756.

36 S. Brenner, Genetics, 1974, 77, 71-94.

37 J. Krijgsveld, R. F. Ketting, T. Mahmoudi, J. Johansen, M. Artal-Sanz, C. P. Verrijzer, R. H. Plasterk and A. J. Heck, Nat. Biotechnol., 2003, 21, 927-931.

38 D. Höglinger, P. Haberkant, A. Aguilera-Romero, H. Riezman, F. D. Porter, F. M. Platt, A. Galione and C. Schultz, eLife, 2015, 4, 1-20.

39 R. S. Kamath, A. G. Fraser, Y. Dong, G. Poulin, R. Durbin, M. Gotta, A. Kanapin, N. Le Bot, S. Moreno, M. Sohrmann, D. P. Welchman, P. Zipperlen and J. Ahringer, Nature, 2003, 421, 231-237.

40 L. Orci, A. A. Like, M. Amherdt, B. Blondel, Y. Kanazawa, E. B. Marliss, A. E. Lambert, C. B. Wollheim and A. E. Renold, J. Ultrastruct. Res., 1973, 43, 270-297. 